\title{
GAMMA-RAY INDUCED DOPPLER BROADENING AND THE DETERMINATION OF LIFETIMES OF EXCITED NUCLEAR STATES
}

\author{
H. B. Borner, J. Jolie, S. J. Rob inson, E. G. Kessler, S. Ullbig, R. F. Casten, \\ S. M. Dewey, G. Greene, R. Deslattes, K. P. Lieb, B. Krusche, \\ and J. A. Cizewski
}

\section{Presented at the \\ 7th International Symposium on Capture Gamma-Ray Spectroscopy Asilomar, California \\ October 14-19, 1990}

\begin{abstract}
DISCLAIMER
This report was prepared as an account of work sponsored by an agency of the United States Government. Neither the United States Government nor any agency thereof, nor any of their employees, makes any warranty, express or implied, or assumes any legal liability or responsibility for the accuracy, completeness, or usefulness of any information, apparatus, product, or p-ocess disclosed, or represents that its use would not infringe privately owned rights. Reference herein to any specific commercial product, process, or service by trade name, trademark, manufacturer, or otherwise does not necessarily constitute or imply its endorsement, recommendation, or favoring by the United States Government or any agency thereof. The views and opinions of authors expressed herein do not necessarily state or reflect those of the United States Government or any agency thereof.
\end{abstract}

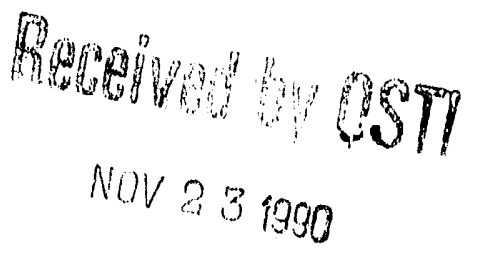




\title{
GAMMA RAY INDUCED DOPPLER BROADENING AND THE DETERMINATION OF LIFETIMES OF EXCITED NUCLEAR STATES
}

H.G. Börner1, J. Jolie1, S.J. Robinson 1, E.G. Kessler2, S. Ulbig3, R.F. Casten4, S.M. Dewey2, G. Greene2, R. Deslattes2, K.P. Lieb3, B. Krusche5, J.A Cizewski6

1) Institut Laue Langevin, F38042 Grenoble, France

2) Nat. Inst. of Standards and Technology, Gaithersburg, MD 20899, USA

3) Universität Göttingen, D3400 Göttingen, Germany

4) Brookhaven National Laboratory, Upton, N.Y.,, 11973, USA

5) Universität Giessen, D6300 Giessen, Germany

6) Rutgers University, Physics Dept, New Brunswick, N.J., 08903, USA

\begin{abstract}
Measurements of lifetimes of excited states in nuclei yield crucial information for sensitive tests of nuclear models. Here a novel method will be discussed which involves the GRID (Gamma Ray induced Doppler broadening) technique, in which Doppler broadening is observed in a transition from a nucleus recoiling from the emission of a previous gamma ray. As the recoil energy is extremely small, ultra-high energy resolving power has to be used. To date all such experiments have been. carried out at ILL using the GAMS4 double flat crystal spectrometer which is operated in a NIST-ILL collaboration.

The method can be used for all lifetimes below a few picoseconds. The wide range of applicability, together with the very exhaustive set of data often obtained, is an advantage with respect to many other metho's. The characteristic features of GRID will be discussed using some selected examples.
\end{abstract}

\section{Introduction}

Nuclear spectroscopy is an important tool which allows us to improve our knowledge of the nuclear many-body problem and the role forces play within the nucleus. Many complementary techniques have been reveloped to elucidate the complex interplay of nucleons and generaliy it is the combination of several of these techniques (for instance radioactive decay, transfer reactions, neutron capture, inelastic scattering, Coulomb excitation, charged particle reactions and others) which allows the construction and interpretation of detailed nuclear excitation schemes.

The neutron capture $\gamma$-ray reaction (in contrast to most other reactions) is known to be non-selective in terms of nuc!ear structure and, nence, 
provides the opportunity to determine rather complete sets of low lying nuclear states. A famous example is the identification of all intrinsic excitations below $\sim 2 \mathrm{MeV}$ in 168Er [1]. Such studies were possible due to the high resolving power obtained with bent-crystal spectrometers installed at the High Flux Reactor of the ILL [2]. Such a combination simultaneously permits good resolution and high sensitivity. The results of such measurements provide energies, intensities and branching ratios of $\gamma$ transitions connecting nuclear states, important ingredients for comparison with nuclear models.

Within the last three years a new tool has been developed, adding to the field of $\gamma$-ray spectroscopy the possibility of measuring the lifetimes of short lived nuclear excited states, populated after neutron capture. This new technique GRID [3] (Gamma Ray Induced Dur ;', 'er broadening) is based on the measurement of the extremely small Doppler shift characterizing a gamma ray emitted from a nucleus which is recoiling due to the prior emission of another gamma ray. In the following we will focus on the status of this technique by discussing its application to some selected nuclei.

\section{The Instrument}

The most accurate method for the determination of gamma-ray wavelengths relies on crystal diffraction from nearly perfect flat crystals of silicon or germanium. The highest precision and resolution, obtained to date, is with a two axis flat crystal spectrometer, GAMS4, installed at the Institut Laue Langevin (ILL), Grenoble, and operated in a NIST/ILL collaboration. The drawback of the extremely low luminosity of such a spectrometer is countered to an acceptable level, by using high mass targets $(\approx 10 \mathrm{~g})$ in combination with a high neutron flux, such as available at the throughgoing beam tube $\mathrm{H} 6-\mathrm{H} 7$ at the ILL high flux reactor $\left(\phi_{n}=5 \times 1014 / \mathrm{cm}^{2} \mathrm{~s}\right)$. Details of this spectrometer are discussed elsewhere [4,5]. Here we will discuss a special application of ultra-high resolution work: the measurement of detailed characteristics of line shapes (dispersion) rather than line centroids (precision). An extremely important characteristic of a two-axis flat crystal spectrometer, with a view to the study of lineshapes, is that it can be operated in both a dispersive and a non-dispersive mode. This is illustrated in Fig. 1 and described in detail in refs. [3,4]. A typical measurement procedure involves placing the first crystal at $\theta$ Bragg and rocking the second crystal (and detector) so that its orientation is $+\theta$ Bragg or $-\theta$ Bragg from the first diffracted beam. Except for very small $(\approx 10-7)$ corrections due to finite vertical divergence, the angular separation of these two diffracted beams is $2 \theta$ Bragg. At $+\theta$ Bragg the two crystals are parallel to each other. This is a non-dispersive geometry (as the crystal planes are parallel to each other, radiation from a broad energy window determined by the solid angle can be reflected simultaneously) and the profile obtained by rocking the second crystal is the response function at angle $\theta$ Bragg. At $-\theta$ Bragg the two crystals are aligned at $2 \theta \mathrm{Bragg}$ with respect to each other. This is a dispersive 
geometry (only exactly one wavelength $\lambda$, within the instrumental resolution, can be successively Bragg-diffracted) and as such one measures the wavelength distribution of the observed $\gamma$-rays.

The diffraction angles are measured by polarization sensitive Micheison interferometers $[4,5]$ which have a sensitivity of $\approx 10-10$ radians. The best instrumental resolution obtained is currently in the order of $\Delta E / E \approx$ 2.10-6 (fig.1)

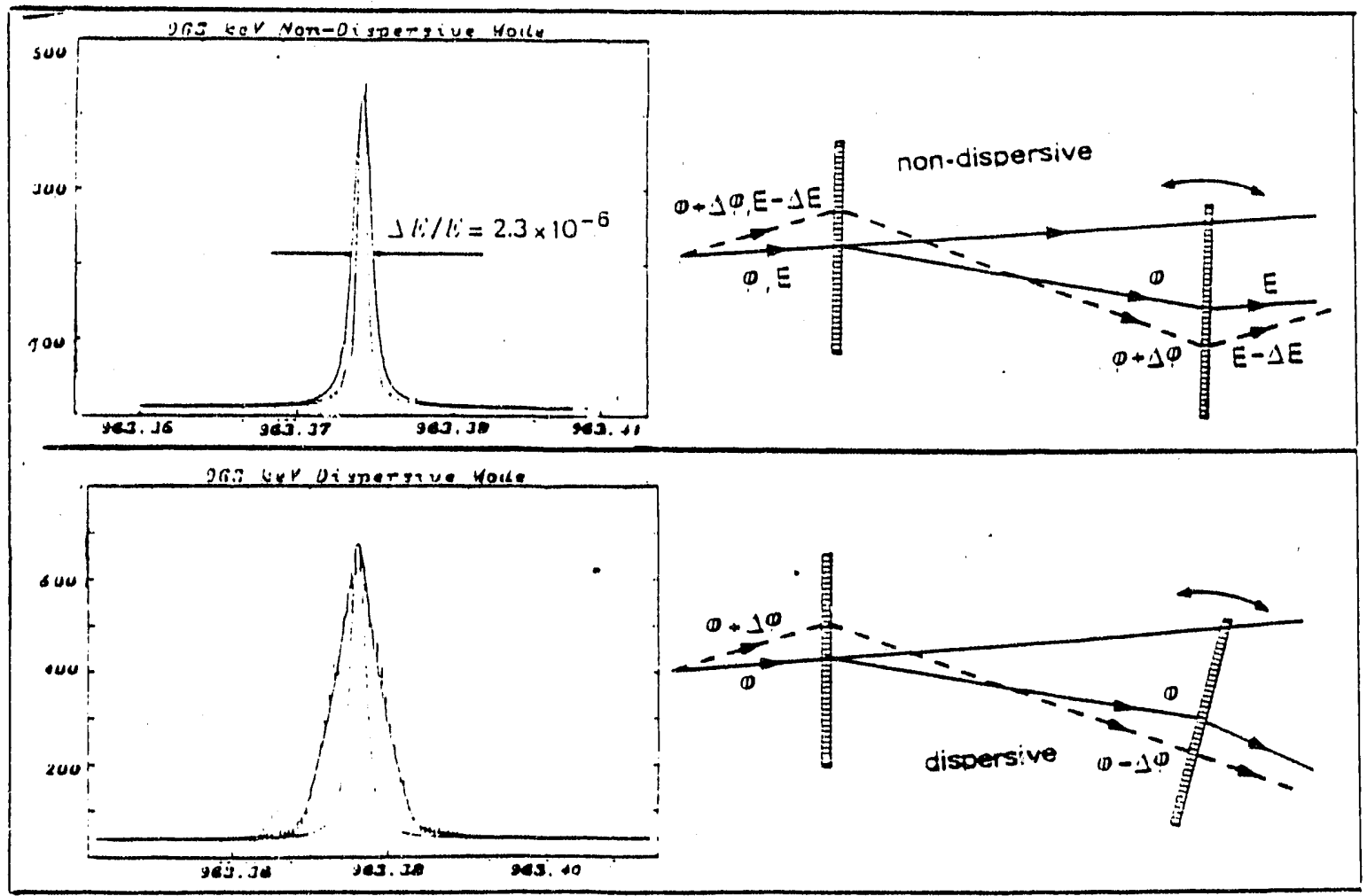

Fig. 1: GAMS4-reflected profiles of a $963 \mathrm{keV}$ transition in $152 \mathrm{Sm}$, emitted after $\mathrm{K}$-capture in 152Eu. In K-capture the nucleus experiences a recoil due to the emission of a neutrino. In this example the recoil is as small as $\approx 3 \mathrm{eV}$. Nevertheless, one can clearly discern the Doppler broadening measured in the dispersive mode (lower part) by comparison with the lineshape obtained in the non-dispersive mode. $A$ resolution of $\Delta E / E \approx 2.10^{-6}$ has been achieved. The dashed line in the non-dispersive data indicates the theoretical lineshape obtained from dynamical diffraction theory calculations for the crystals used ( $\mathrm{Si}, 660$ planes, $2.5 \mathrm{~mm}$ thickness). The resolution obtained to date is very close to this theoretical limit. The dashed line in the dispersive profile represents again the instrumental response, demonstrating the amount of broadening observed. The spectral distribution of the $\gamma$-transition can be obtained by deconvolution of the non-dispersive scan from the dispersive one. 


\section{The GRID-Method}

For several decades one of the techniques used to study the lifetimes of excited nuclear states has been the Doppler-shift attenuation method (DSAM) [6,7 and refs. therein]. In reactions where accelerated heavy ions interact with target nuclei in a solid medium, the subsequent reaction products can recoil with typical velocities of the order of 0.1 to $10 \%$ of the velocity of light. The Doppler shift of gamma rays emitted by the recoil products can be up to several $\mathrm{keV}$ and is comparable to the resolution obtained with solid-state detectors. As the shift is attenuated when emission occurs as the nucleus slows down, lifetimes of excited states can then be obtained by comparison with the slowing down time of the recoiling ions in the target. In these reactions one neglects the recoil imparted to the nucleus due to the emission of gamma rays as this leads to recoils which are neglegible with respect to the original recoil velocities. However, in the GRID method it is exactly this small recoil which gives the Dopoler broadening we observe, using ultra-high resolution spectroscopy after thermal neutron capture.

When the neutron capture state, at energy $E_{C}$, decays via the emission of $\gamma$ rays this induces a recoil $\left(v / c=E \gamma / \mathrm{mc}^{2}\right)$ which leads to velocities $v_{R} / c \approx$ $10-4$ to $10-6$. The neutron energy of $\approx 25 \mathrm{meV}$ can be neglected. As these gamma-rays are isotropically emitted this leads to an isotropic distribution of the recoil directions and hence not a Doppler shift, but a Doppler broadening is observed.

The observed Doppler-broadened profile is a function of three separate conditions:

i) the velocity of the recoiling atoms at the moment when the level of interest is populated

ii) the time differential behaviour (slowing down) of the recolling atoms

iii) the lifetime of the nuclear state which deexcites by the emission of the $\gamma$ ray being observed.

The principle of the GRID technique is that, provided i) and ii) are known, lifetimes can be extracted.

Fig. 2 summarizes qualitatively different scenarios from which the influence of the velocity distributions (emerging from different population routes) on the expected Doppler profiles can be seen, illlustrating point $i$.

In all cases we start with the capture of a thermal neutron. The capture state then decays via different routes to an excited state $E_{X}$, the lifetime of which we want to measure by determining the Doppler profile of one of its depopulating transitions E $\gamma$. In the simplest case (fig. 2a) the capture state feeds $E_{x}$ with one primary transition of energy $E_{p}$ and the nucleus will then recoil with a velocity:

$$
V_{R}=E_{p} / M c
$$



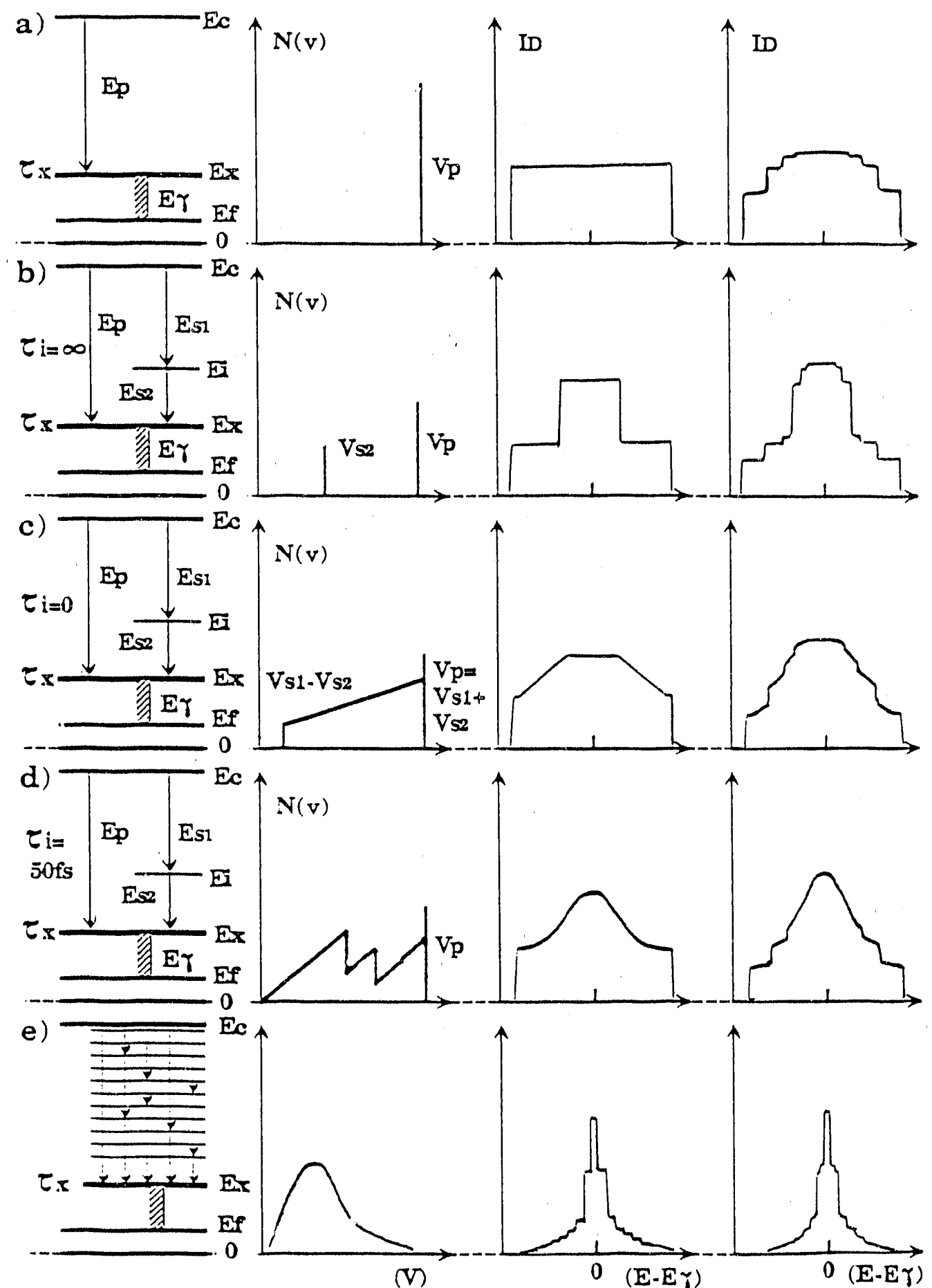

Fig. 2: Five feeding scenarios: Row a): Primary feeding only. b): Two-step cascade with intermediate lifetime $\tau_{i}=\infty$. c) Two-step cascade with intermediate lifetime $\tau_{i}=0$. d) Two-step cascade with finite intermediate lifetime. e) Statistical cascade feeding. $\tau_{i}$ corresponds to the lifetime of the level $E_{i} ; v_{p}, v_{s} 1$ and $v_{S 2}$ correspond to the velovity $v / c=E / M c 2$, induced by the emission of a $\gamma$ ray with energy $E_{p}, E_{s 1}$ and $E_{s 2}$, respectively. 
Then the energy E $\gamma$ will be Doppler shifted by:

$$
\Delta E_{D}=E_{\gamma} \cdot V_{R} / c=\left(E_{\gamma} \cdot E_{p} / M c^{2}\right) \cos \phi
$$

where $\phi$ is the angle between directions of observation and recoil. The maximum Doppler shift will be

$$
\Delta E_{D^{m}}=E_{\gamma} \cdot E_{p} / M c^{2}
$$

Integrating over all projections we obtain the predicted Doppler profiles. For an infinitely well defined axis of observation, and no slowing down, this leads to a Doppler broadened intensity distribution of:

$$
\begin{aligned}
& \text { ID }(E, v)=\text { const, for }\left(E_{\gamma} \Delta E_{D}\right)<E<\left(E_{\gamma}+\Delta E D\right) \\
& I D(E, v)=0, \quad \text { elsewhere }
\end{aligned}
$$

For the case of the two-axis flat crystal spectrometer, with its extremely precise definition of the axis of observation, it only remains to fold these profiles with the instrumental response at $E_{\gamma}$, which has been determined in a non-dispersive scan. The profile discussed above is obtained for a freely flying atom.

However, the recoiling nuclei are slowed down in the target material, and this allows us to compare the slowing down time and the lifetime of the excited state $E_{X}$ :

(5) $\Delta E_{D}(t)=E_{\gamma \cdot v(t) / c}$

where $v(t)$ depends on the slowing down process. The decaying intensity of the state $E_{X}$ at $v(t)$ is a function of the mean time interval between population and decay of this state and, as such, establishes the link between the slowing down time and the lifetime $\tau_{x}$.

The stopping theory necessary to describe the slowing down depends on detailed information concerning structure and composition of the target material and, hence, the interatomic potentials. Moreover, different recoil velocities might induce completely different mechanisms in the slowing down process. These aspects are reviewed in detail in other contributions $[8,9,10]$.

For the purpose of the discussion here the simplest approach is to approximate the slowing down by discrete binary collisions of the recoiling atoms with other atoms in the bulk of the target (due to the low recoil energy, quantum mechanical scattering effects can be neglected and 'nuclear' scattering is largely dominant). This leads to Doppler profiles summed over the different velocity contributions, which are obtained after collisions with neighbouring atoms: 
(6)

$$
\begin{aligned}
I D(E)= & I_{s}(E) \exp \left(-\sum_{k=0}^{N-1} \frac{t_{k}}{\tau}\right)+\sum_{n=1}^{N} I D\left(E, v_{n-1}\right) x \\
& x\left[\exp \left(-\sum_{k=0}^{n-2} \frac{t_{k}}{\tau}\right)-\exp \left(-\sum_{k=0}^{n-1} \frac{t_{k}}{\tau}\right)\right],
\end{aligned}
$$

where $v_{k}=v_{R} / 2 k / 2$ is the velocity after the $k$-th collision and $t_{k}=r_{k} / v_{k}$ is the time between the collisions $(k-1)$ and $k$. The fraction $I_{S}(E)$ describes the "stopped peak" due to thermalized atoms [8], while ID (E, $\left.v_{n}\right)$ describes the Doppler profile after the $n$-th collision. The measured line profiles are then obtained by convolution with the instrumental response function.

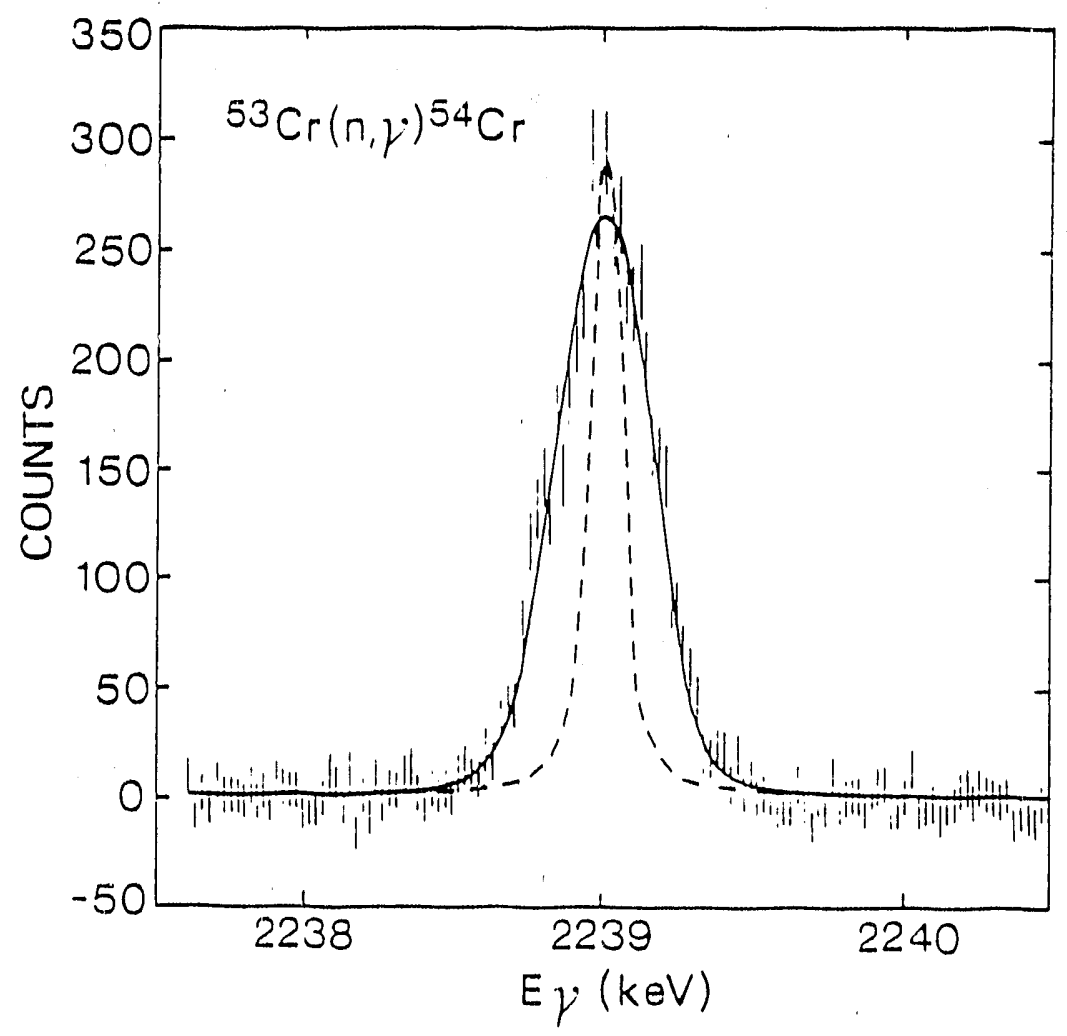

Fig. 3: Doppler-broadened lineshape measured for the transition $3074 \rightarrow 835$ $\mathrm{keV}$ in $54 \mathrm{Cr}$. The dasined ine shows the spectrometer response function, the solid line the fitted lineshape. 


\section{Examples}

\section{1) Primary Feeding}

In the example considered above, the level of interest is uniquely fed by one $\gamma$-ray (primary). This results in a clearly defined original recoll velocity $\checkmark R / C=E_{p} / M c 2$. For the first lifetime measurements [3, 11-13] carried out using GRID, nuclei have been chosen where the levels of interest were fed, to a good approximation, by known, simple patterns. In sucin a case complications due to unknown feedings and time delays caused by cascade feeding via intermediate states are small, thus allowing a thorough test of the method under transparerit experimental conditions. An example of such a measurement is given in ref. [11] where lifetimes of some specific levels in ${ }^{54} \mathrm{Cr}$ have been studied, yielding evidence for a mixed symmetry state at $3(74 \mathrm{keV}$. Fig. 3 shows an example of the line profile obtained for the decay of this $23+$ level via the $2239 \mathrm{keV}$ transition to the $21+$ state. For the first time a definite lifetime (13(2)fs) was deduced for this level. The figure demonstrates the sensitivity of GRID in this time region. (The characteristics of mixed symmetry states in nuclei near closed shells are reviewed in more detail by $S$. Robinson et al. in another contribution to this conference).

\section{1) Cascade Feeding}

The case of predominant primary feeding, however, is not the standard case in $(n, \gamma)$, especially in heavier nuclei. More typically, cascade feeding contributes to - or even dominates - the population of a low lying state. The resulting velocity distribution of the recoiling atoms depends on the energies of all feeding transitions and the lifetimes of the respective intermediate levels.

Fig. 2 summarizes some of the most important scenarios which may be involved in different feeding patterns. Starting with the case of predominant primary feeding as discussed above, fig. $2 e$ represents the case of pure statistical population. For each case the corresponding velocity distributions (column 2) and corresponding Doppler profiles (column 3,4) are deduced. Column 3 represents the case for a treely flying particle, which is only observed for very short lifetimes $(\approx 1 \mathrm{fs})$, where the level decays before any slowing down can take place. Column 4 includes, qualitatively, slowing down. This comparison stiuws that the measured Doppler profiles are quite sensitive to the original velocity distributions.

A model case in $49 \mathrm{Ti}$, representative of the situation cited above, is discussed in another contribution to this conference [14]. In this case the decay of the capture state follows a scenario as depicted in fig. 2d. A primary $\gamma$ transition feeds (to almost $100 \%$ ) the $3 / 2-$ level at $3260 \mathrm{keV}$, wilich in turn decays, via the $1498 \mathrm{keV}$ transition, to the $1762 \mathrm{keV}$ state. We have measured the lifetime of the $3260 \mathrm{keV}$ level to be 17(2) fs. As described in 
detail in reference [14] one can determine the lifetime of the $1762 \mathrm{keV}$ state as a function of i) the population of the $3260 \mathrm{keV}$ level, ii) the lifetime of the latter and iii) the energy of the directly preceeding transition (1498 keV), as these three parameters allow the determination of the original recoll velocity distribution (including the time differential slowing down) for the decay of the $1762 \mathrm{keV}$ level.

In a more general case one might not know the detalls of the feeding paths. Can they be reconstructed, and to what extent? To test this, let us assume that we do not know any of the feeding characteristics of the 1762 $\mathrm{keV}$ level. Now we fit the measured $1762 \mathrm{keV}$ Doppler profile as a function of a single initial recoll velocity $\mathrm{V} / \mathrm{c}$ (this corresponds to the scenario described in fig. $2 b)$. We then analyse the respective $\chi 2$ obtained as a function of $v / c$ (fig. 4, curve 1). As the Doppler profiles are sensitive to the recoil velocity, we can correlate the quality of the fit to the velocity distribution $N(v)$. The result is shown in curve 2 of fig. 4 by requiring $N(v) \propto 1 / \chi 2$ (and normalizing arbitrarily) Comparison with the velocity distribution calculated from the known decay pattern (curve 3 , fig. 4) [14] shows a striking similarity. The mean life of the $1762 \mathrm{keV}$ level, deduced with the known feeding, is 55(5) fs. Using the velocity distribution which results from the shape analysis one obtains $\sim 60$ fs, In good agreement. Such a prócedure might allow, in a qualitative way, to test the feeding assumptions and to verify whether or not important paths are missing.

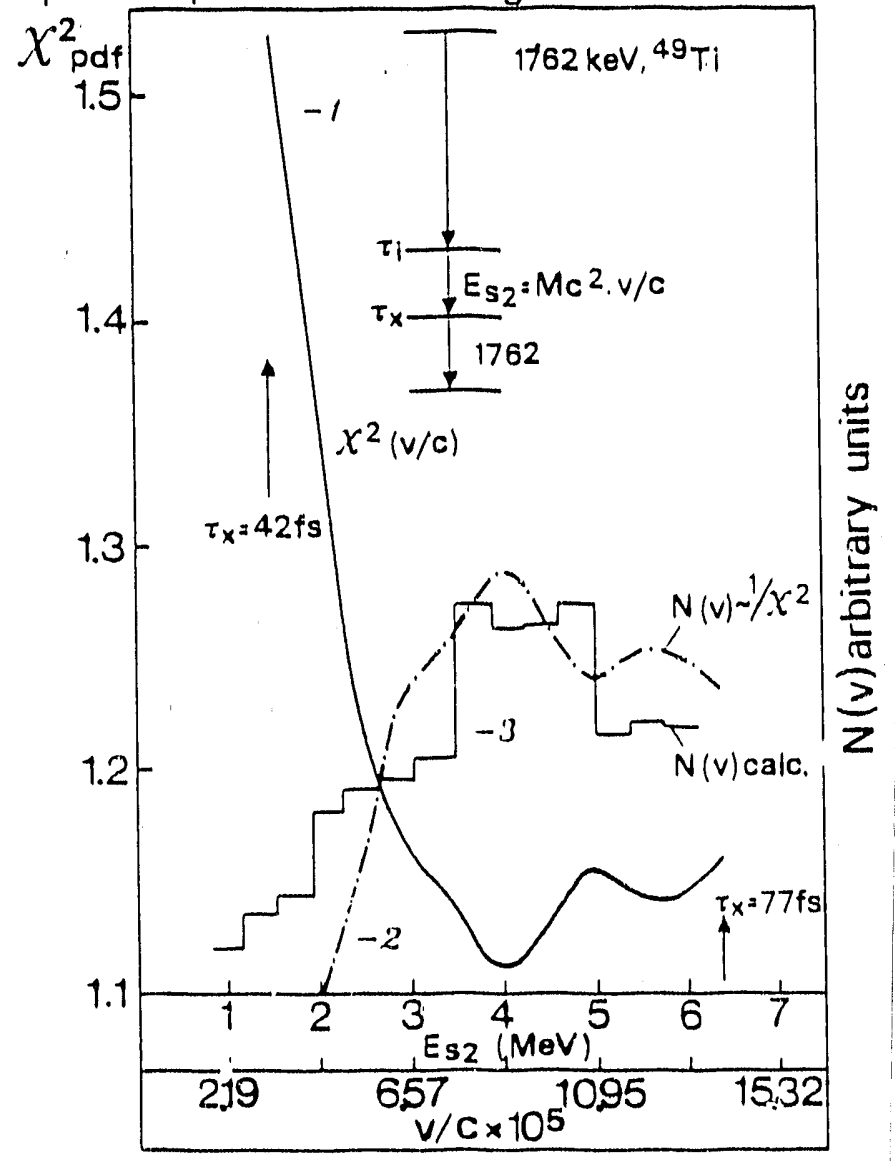

Ela 4: Investigation of the velocity distribution in a two-step cascade in $49 \pi$, assuming unknown feeding of the $1762 \mathrm{keV}$ level. $N(v)$ is normalized in an arbitrary manner and therefore reflects a qualitative picture only of the velocity distribution ! 


\section{Iii) Statistical Feoding}

As mentioned above, cascade feeding procedes via more complex feeding patterns where many intermediate states are involved (fig. 2e). The extension of the treatment from one to many intermediate levels is technically straight forward - provided the respective energies and lifetimes are known. This, however, is generally not the case.

The experimentally missing population strength and the lifetimes of the intermeciate states can be approximated by using a statistical model approach. A Monte Carlo code has been developed [15] to simulate these cascades and to deduce the recoil velocity distributions. The level densities are obtained using either a Constant Temperature Fermi gas approach (CTF) $[16,17]$, or the Bethe jarametrisation $[16,17]$. The E1, M1 and E2 strengths are obtained using a giant resonance-ansatz. The level distance distribution follows a Wigner distribution (for flxed spins and parities).

Whereas most statistical features depend strongly on the decay branchings, the time development is dictated by the decay widths. The recoil distributions are therefore quite sensitive (see fig. $2 b-d$ ) to the $\gamma$-ray strength functions and may - in turn - provide a method to test them. This, however, will be a rather longstanding effort. An example in $144 \mathrm{Nd}$ is described below.

The level scheme of $144 \mathrm{Nd}$ had recently been extensively studied [18] by using the ILL-bent crystal spectrometers. We discuss, in the following, one speciflc state, the $2+3$ level at $2.073 \mathrm{MeV}$. The experimental knowledge concerning ieeding and decay of this level is summarized in fig. 5a. It appears that $\approx 85 \%$ of the feeding strength has not been observed. This part is simulated by a statistical distribution using the CTF approach. From the measured Doppler profile of the depopulating $1377 \mathrm{keV}$ transition one obtains a mean life $\tau \cong 145+40$ - 30 fs.

This result is in disagreement with a previous resonance scattering experiment [19] which gave 60(30) is. If we now wanted to reproduce this latter result we should have to replace the statistical model approach by extreme feeding assumptions with a peaked velocity distribution which corresponds to an energy $E_{S 2}$ of $\approx 1.2 \mathrm{MeV}$. Fig. $5 \mathrm{~b}$ shows, in a similar way to the $49 \mathrm{Ti}$ case, the $\chi 2$ variation as a function of $\mathrm{v} / \mathrm{C}=\mathrm{E}_{\mathrm{S} 2} / \mathrm{Mc}^{2}$ and as a function of the deduced lifetime. A lifetime as reported in [19] is incompatible with the above shape analysis. $\tau \neq 60$ fs would require a $\chi 2$ minimum at the corresponding energy. Additionally this is ruled out by the experiment where no strong unplaced $\gamma$-transitions are observed in the appropriate energy window. Again, the same interesting feature as observed above emerges from the $\chi 2$ analysis as a function of the variation of $v / c$ : One finds a velocity distribution $N(v)$, which, in this case, is very close to the one calculated from a statistical approach (fig. 5c) and this, inherently, can be seen as a justification of the application of the statistical feeding approach for this state. It should be stressed again that such an analysis is possible due to the very specific shapes of the Doppler profiles. From the slope in fig. $5 b$, one sees 


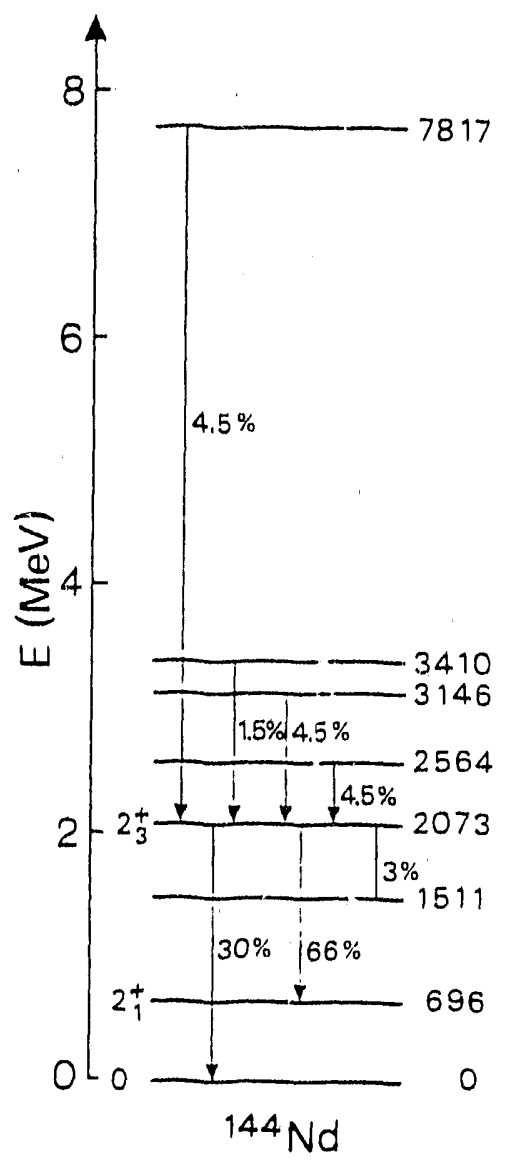

Eig.5a:Feeding and decay of the $2073 \mathrm{keV}, 2+3$ level in $144 \mathrm{Nd}$

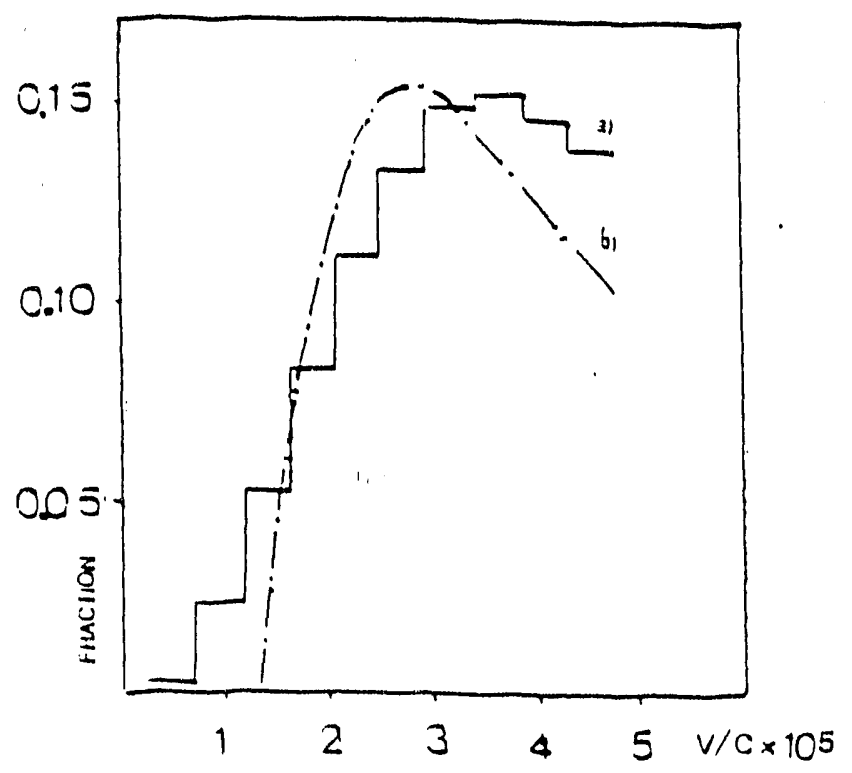

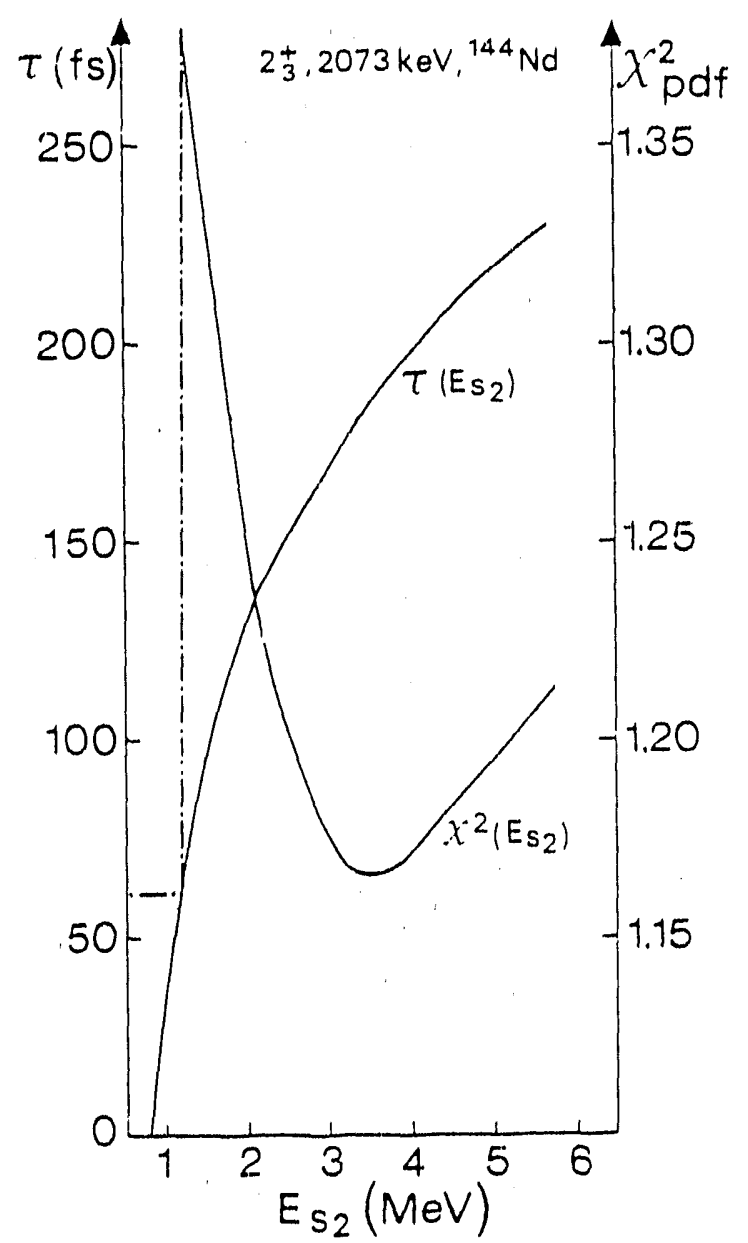

Eig. 5b: Shape analysis for the $1377 \mathrm{keV}$ transition in $144 \mathrm{Nd}$

Fig. 5c: Comparison of velocity distributions obtained from

a) a statistical calculation

b) the shape analysis, normalized arbitrarily. 
that details of feeding enter more strongly into the Doppler profiles at shorter lifetimes. This leads to two consequences:

a) Uncertainties in the feeding description are less important for longer lifetimes and consequently

b) detailed tests of statistical models can better be done at short lifetimes!

A third case of a reconstruction of the feeding is shown in fig. 6 for a 3-, $2022 \mathrm{k} \vartheta \mathrm{V}$ state in $168 \mathrm{Er}$. The shape analysis $(\chi 2)$ as a function of $\mathrm{v} / \mathrm{c}$ yields a recoil distribution $N(v) \propto 1 / \chi 2$ which peaks at the velocity which corresponds to essentially primary feeding. Again, this is in agreement with the experiment [1], where one deduces a primary feeding strength of $60 \%<1 p<95 \%$ for this level.

To summarize, we have shown that careful shape analysis of GRIDprofiles allows the identification of trends in the original recoil distribution which, in turn, are a function of the feeding pattern. These distributions can be compared to those obtained from other inputs, like for instance a statistical model approach. Detailed investigations of this kind are currently in progress. In the examples shown before (fig. $4,5,6$ ) we have also plctted the variation of $\tau$ as a function of $v / c$. One can see that, although the Doppler

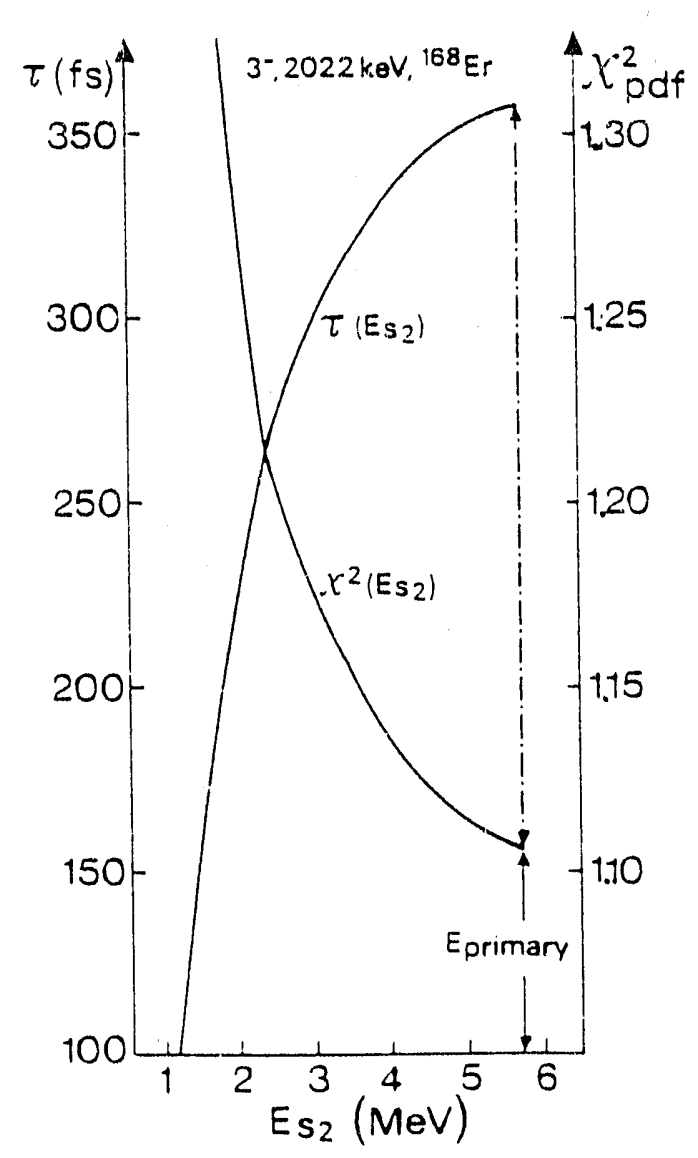

profiles are very sensitive to the recoil distributions, the $\tau$-values deduced for a given $v / c$ vary in relatively restricted regions, e.g. even for extreme limits' of $\mathrm{v} / \mathrm{c}$, corresponding to fig'2b and $2 \mathrm{c}$, significant lifetime limits can be deduced. A scenario following fig, $2 b$ leads to a low limit on $\tau$ (deperiding on the choice of Es2); $2 \mathrm{c}$ leads likewise to an upper limit.

Eig. 6. Shape analysis of the $1942 \mathrm{keV}$ transition in, 168Er. 


\section{IV) GRID in "heavy nuclei"}

In the region of light nuclei many of the lifetimes of levels which can be studied with GRID, can also be investigated using DSAM - at least as long as the lifetimes are not very short. In heavier nuclei, close to or at stability, the situation is different and, generally, only relatively few lifetimes are known, very often studied from Coulomb excitation measurements. Here GRID offers the possibility for more extended studies.

\section{A) $196 \mathrm{Pt}$}

$196 \mathrm{Pt}$ was the first nucleus whose level scheme was interpreted in terms of the $O(6)$ limit of the IBA. It was found that the agreement with predicted $0(6)$ E2 selection rules and branching ratios is excellent. One critical test however, has been lacking to date: The measurement of any absolute transition rates (or limits) for the decay of $\sigma<N$ states, and especially for the $0+$ "bandhead" of these families. Fig. 7a shows the experimentally observed decay of the $\mathrm{O}_{3}+, 1402 \mathrm{keV}$ state (which belongs to the $c=4, \tau=0$, group) to the $21+$ level of the $\sigma=N=6, \tau=1$, group in 196pt. In $0(6)$, the selection rules are $\Delta \sigma=0, \Delta \tau= \pm 1$ and therefore the decay of the

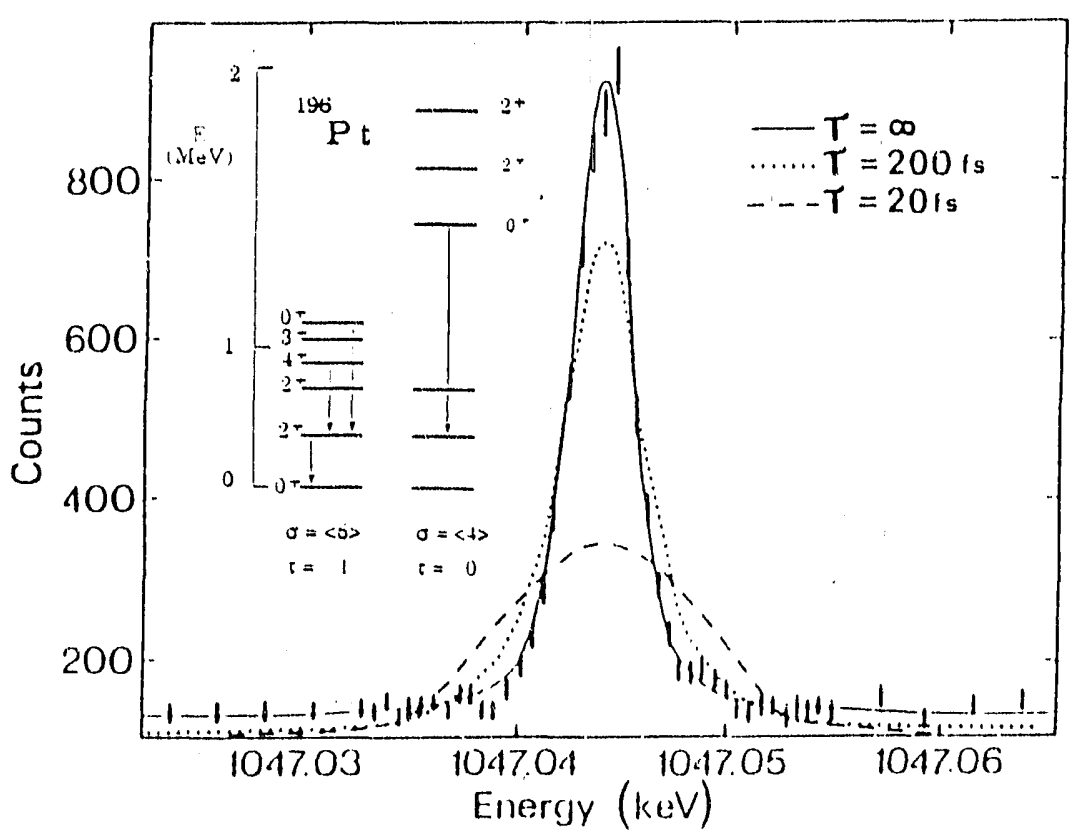

Eig. 7. The Doppler profile observed for the $1047 \mathrm{keV}$ transition in $196 \mathrm{Pt}$, in comparison with the predicted shapes for $\tau=\infty$ ( unbroadened shape), 200 is and $20 \mathrm{fs}$. It is evident that the peak does not show broadening and thus the lower limit of $\tau>1.86 \mathrm{ps}$ is obtained. 
$\mathrm{O}_{3}+$ state should be strongly hindered. The observed decay preserves the stronger $\tau$ selection rule $(\tau=0 \rightarrow \tau=1)$ and violates the $\sigma$-selection rule. We have obtained results for lifetimes of several levels in 196Pt [20]; however, the principal aim was to obtain a value or limit for the lifetime of the $1402 \mathrm{keV}$ level which decays predominantly by the $1047 \mathrm{keV}$ transition to the $21^{+}$ state. Fig 7 shows the data for the $1047 \mathrm{keV}$ transition. A lower limit of $\tau>1.86$ ps is obtained under the assumption of extreme side feeding, following Fig. $2 \mathrm{~b}$ with $E_{s} 2$ corresponding to the highest known secondary feeding transtion. This lifetime limit yields : $B\left(E 2: 0_{3}+-21^{+}\right) \leq 0.034 e^{2 b}$ [20]. This result is in agreement with an order of magnitude hindrance for the $\sigma$ forbidden transition in the $O(6)$ limit, since allowed $B(E 2)$ values in $0(6)$ are typically $\approx 0.4 e^{2} \mathrm{~b} 2$. This provides strong support for the validity of the $\sigma$ selection rule. Simultaneously it definitely rules out the interpretation of this level in the $U(5)$ limit which predicts a decay 13 times faster than the obtained limiting value.

\section{B) $168 \mathrm{Er}$}

For several decades a significant issue in the nuclear structure of deformed nuclei has beeri whether or not two-phonon collective excitations, such as double gamma vibrations, exist. To date there exist different theoretical approaches treating this problem, which have reached different conclusions. The nucleus $168 \mathrm{Er}$ offers an excellent opportunity to test these different models: The 2+ bandhead of the $\gamma$ vibration lies at the low energy of $821 \mathrm{keV}$, so that the two-phonon gamma vibration can occur in a relatively clean part of the spectrum. From an extensive earlier study [1] one knew that the lowest possible candidate for a two-phonon $\kappa^{\pi}=4^{+}$gamma vibration was the 4+ bandhead at $2.055 \mathrm{MeV}$. This is the lowest lying intrinsic $\mathrm{K} \pi=4+$ excitation and has also the expected energy and decay properties of a dominant E2 branch to the single phonon $\gamma$-vibration. However, the absolute strength, $B\left(E 2 ; 4^{+} \rightarrow 2+\gamma\right)$, was unknown.

Therefore we have measured the lifetimes of the $4+$ and $5+$ members of this band [21], at 2055 and $2169 \mathrm{keV}$, respectively, by measuring the Doppler broadening of the transitions of $1234 \mathrm{keV}(4+\rightarrow 2+\gamma)$ and $1277 \mathrm{keV}$ $\left(5^{+} \rightarrow 3+\gamma\right)$. Fig. 8 shows a summation over 10 individual scans for the $1234 \mathrm{keV}$ transition. The data are also compared to the predicted lineshapes for $\tau=1$ ps and $\tau=40$ fs. From the limits for the lifetimes of the $4+$ and $5^{+}$ levels, we obtain $0.014 \mathrm{e}^{2 \mathrm{~b} 2}<\mathrm{B}\left(\mathrm{E2} 24^{+} \rightarrow 2+\gamma\right)<0.041 \mathrm{e}^{2 \mathrm{~b} 2}$ and 0.017 $e^{2} b^{2}<B\left(E 2: 5^{+} \rightarrow 3+\gamma\right)<0.090 e^{2} b^{2}$, respectively. These values are of the same order of magnitude as the known value of $B(E 2: 2+\gamma \rightarrow 0+)=0.0264(9)$ $e^{2} b^{2}$ and thus clearly demonstrate that these transitions are collective in 
nature. These results show, for the first time, definite evidence for the existence of two-phonon vibrational states in a deformed nucleus.

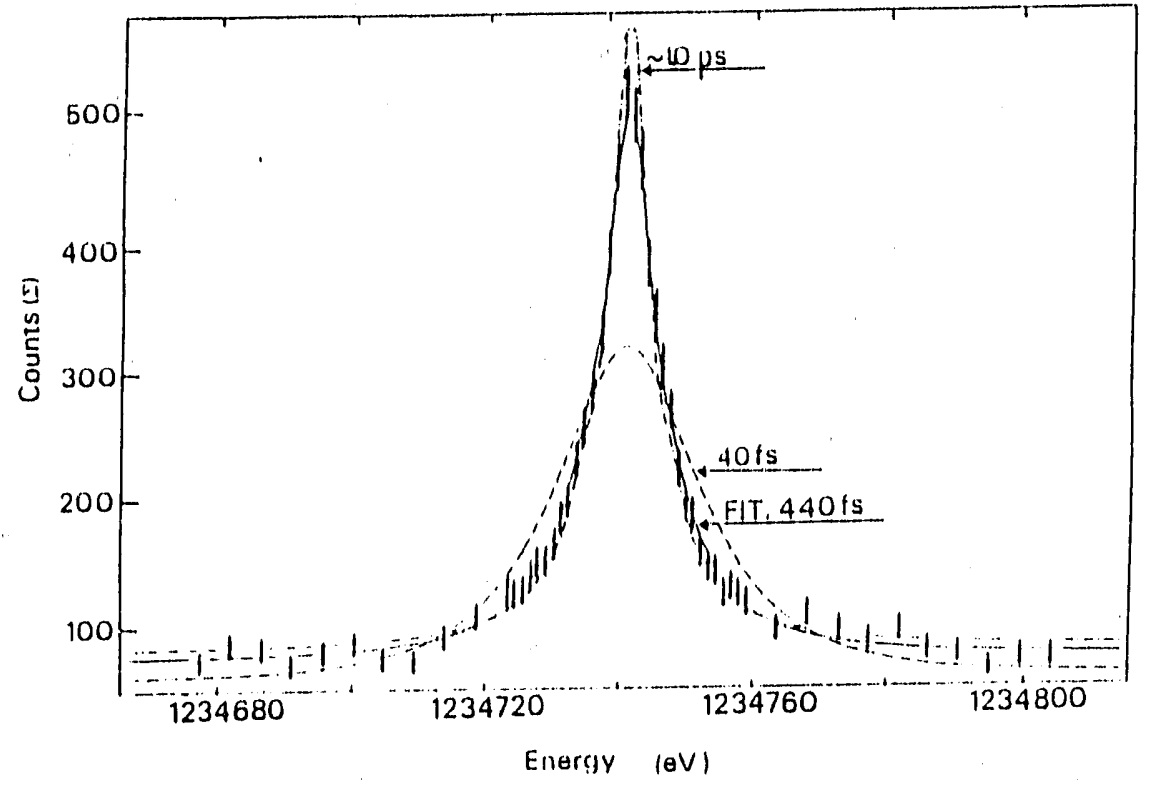

Eig. 8. The Doppler profile measured for the $1234 \mathrm{keV}$ transition in $168 \mathrm{Er}$, shown together with the predicted shapes (dashed lines) for $\tau=10 \mathrm{ps}$ and $\tau=40$ fs.

\section{Conclusion}

We have reviewed a new method to measure lifetimes of excited nuclear levels, which uses Gamma Ray Induced Doppler-broadening, obtained after neutron capture and the successive decay of excited states. As the recoil energy is extremely small ultra-high energy resolving power has to be used. To date all such experiments have been carried out at the Institut Laue Langevin using the GAMS4 double flat crystal spectrometer which is operated in a NIST/ILL collaboration. The method can be used for all lifetimes below several picoseconds. It is accessible to all nuclei, throughout the nuclear chart, which can be reached by neutron capture. In cases, where the feeding path has to be simulated by a statistical approach one profits from the fact that the data are not overly sensitive to the details of the statistical model feeding (which in turn complicates the task, if one wants to test these statistical models). Even the limits derived from extreme feeding assumptions define a region of lifetime values which still allows definitive nuclear structure interpretations.

Work has been supported in part under cotract No. DE-AC02-76CH-00016 


\section{References}

[1] W.F. Davidson, D.D. Warner, K. Schreckenbach, H.G. Börner, J. Simic, M. Stojanovic, M. Bogdanovic, S. Koicki, W. Gelletly, R.F. Casten, G.B. Orr and M.L. Stelts, J. of Phys. GZ, 455, 843 (1981)

[2] H.R. Koch, H.G. Börner, J.A. Pinston, W.F. Davidson, R. Roussille, J.C. Faudou, O.W.B. Schult, Nucl. Inst. Meth., 175, 401 (1980)

[3] H.G. Börner, J. Jolie, F. Hoyler, S. Robinson, M.S. Dewey, G. Greene, E. Kessler, R.D. Deslattes, Phys. Lett. B215, 45 (1988)

[4] M.S. Dewey, E.G. Kessler, G.L. Greene, R.D. Deslattes, H.G. Börner, J. Jolie, Nucl. Inst. Meth. A284, 151 (1989)

[5] E.G. Kessler, G.L. Greene, R.D. Deslattes, H.G. Börner, Phys. Rev. C32, 374 (1985)

[6] P.J. Nolan and J.F. Sharpey-Schaffer, Rep. Prog. Phys. 42. 1 (1979)

[7] J. Keinonen, in: Proc. 5th Int. Symp. of Capture gamma-ray spectroscopy AIP Cnnf. Proc., Vol 125, 557 (1984)

[8] J. Jolie, H.G. Börner, S.J. Robinson, Arialysis of Recoil Doppler piofiles obtained with Ultra High Resolution $(n, \gamma)$-spectroscopy, contribution to this conference

[9] J. Jolie, S. Ulbig, H.G. Börner, K.F. Lieb, S.J. Robinson, P. Schillebeeckx, E.G. Kessler, M.S. Dewey, G.L. Greene, Europhys. Lett. 10_231 (1989)

[10] A. Kuronen, submitted to Phys. Lett, and $J$. Keinonen, contribution to this conference

[11] K.P. Lieb, H.G: Börner, M.S. Dewey, J. Jolie, S.J. Robinson, S. Ulbig, Ch. Winter, Phys. Lett. B215, 50 (1988)

[12] S. Ulbig, K.P. Lieb, Ch. Winter, H.G. Börner, J. Jolie, S.J. Robinson, P.A. Mando, P. Sona, N. Taccetti, M.S. Dewey, J.G.L. Booten, F. Brandolini Nucl. Phys. A505 193 (1989)

[13] S. Ulbig, K.P. Lieb, H.G. Börner, J. Jolie, S.J. Robinson, J.G.L. Booten, M.S. Dewey and Ch Winter, XXV Zakopane School on Physics (1990), World Scientific, in press

[14] S. Ulbig, J. Jolie, H.G. Börner, M.S. Dewey, K.P. Lieb, S.J. Robinson, "Grid lifetime study of the $1762 \mathrm{keV}$ state...", contribution to this conference

[15] B. Krusche et al., to be published, and contribution to this conference

[16] T. v. Egidy, H.H. Schmidt and A.N. Behkami, Nucl. Phys. A481, 109 (1988)

[17] B. Krusche and K.P. Lieb, Phys. Rev. C34, 2103 (1986)

[18] S.J. Robinson et al. , to be published

[19] F.R. Metzger, Phys. Rev., 187,1700 (1969)

[20] H.G. Börner, J. Jolie, S.J. Robinson, R.F. Casten, J.A. Cizewski, Phys. Rev., in press

[21] H.G. Börner, J. Jolie, S.J. Robinson, B. Krusche, R. Piepenbring, R.F. Casten, A. Aprahamian, J. Draayer, submitted to Phys. Rev. Lett. 

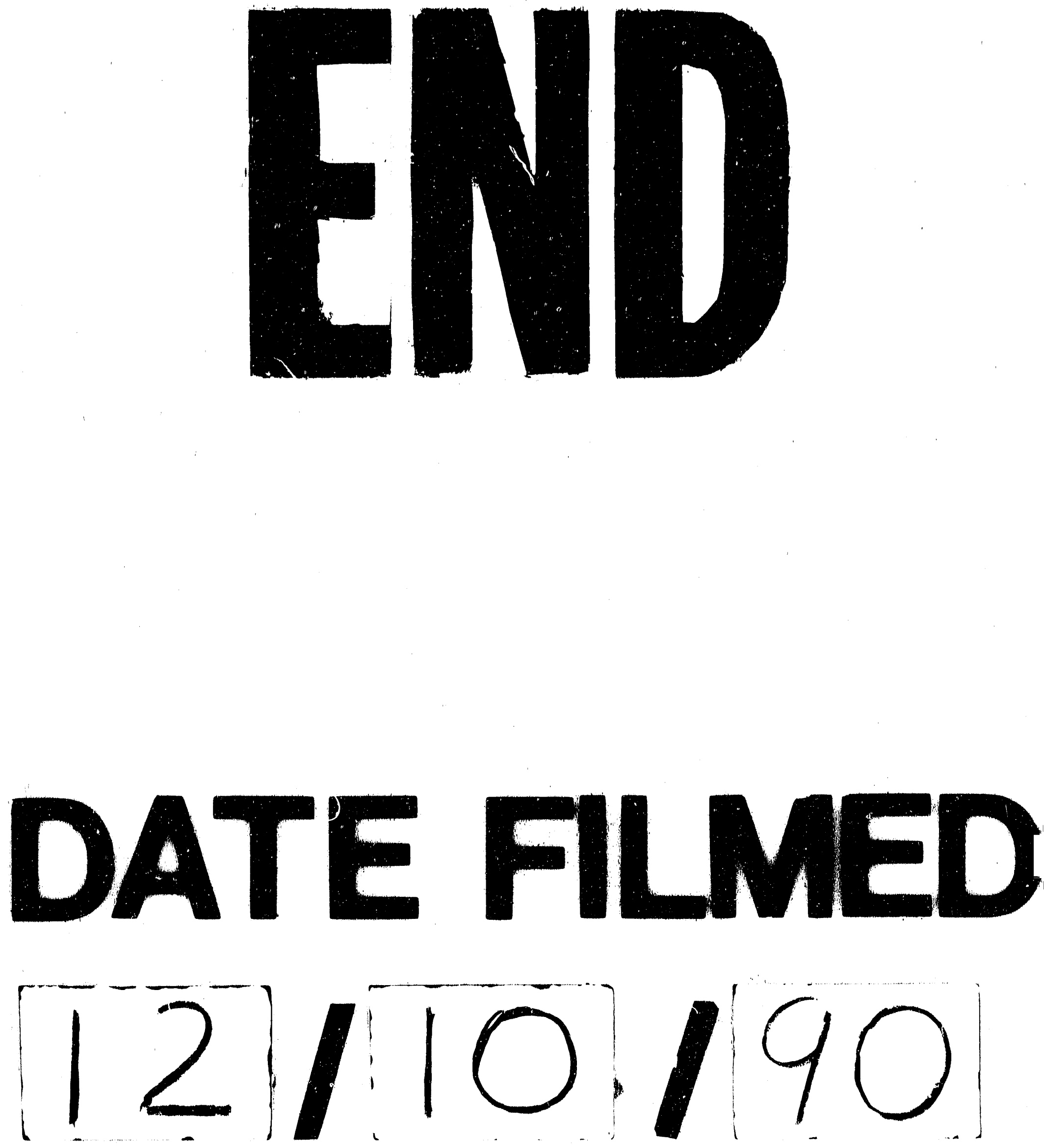
